\title{
Dynamin 3 Gene as a Tumor Suppressor and an Independent Predictive Factor of Poor Prognosis in Patients with Gastric Cancer
}

\section{Ru-Hong Tu}

Fujian Medical University Union Hospital

Jian-Xian Lin

Fujian Medical University Union Hospital

Jian-Wei Xie

Fujian Medical University Union Hospital

Jia-Bin Wang

Fujian Medical University Union Hospital

Jun Lu

Fujian Medical University Union Hospital

Qi-Yue Chen

Fujian Medical University Union Hospital

Long-Long Cao

Fujian Medical University Union Hospital

Mi Lin

Fujian Medical University Union Hospital

\section{Ze-Ning Huang}

Fujian Medical University Union Hospital Ju-Li Lin

Fujian Medical University Union Hospital Hua-Long Zheng

Fujian Medical University Union Hospital

Ping Li

Fujian Medical University Union Hospital

Chao-Hui Zheng

Fujian Medical University Union Hospital

Chang-Ming Huang ( $\nabla$ hcmlr2002@163.com )

Fujian Medical University Union Hospital 
Keywords: Gastric cancer, Dynamin 3, tumor suppressor, immunosuppression

Posted Date: December 22nd, 2021

DOI: https://doi.org/10.21203/rs.3.rs-1072813/v1

License: (c) (1) This work is licensed under a Creative Commons Attribution 4.0 International License. Read Full License 


\section{Abstract}

Gastric cancer is a leading cause of death from malignant tumors worldwide. With the development of genome sequencing technology, an increasing number of key driver genes and tumor suppressors have been discovered. Some studies have suggested that Dynamin 3 (DNM3) is a novel tumor suppressor; however, the role of DNM3 in malignancy remains unclear. We performed a systematic analysis using The Cancer Genome Atlas Stomach Adenocarcinoma (TCGA-STAD) cohorts, and 160 patients with stomach adenocarcinoma at Fujian Medical University Union Hospital (FJMUUH) (48 quantitative PCR [qPCR] and 112 immunohistochemistry). DNM3 expression was found to be downregulated in gastric cancer compared to that in paraneoplastic tissue. Low expression of DNM3 was mainly associated with DNM3 promoter hypermethylation status. Low expression of DNM3 can upregulate the tumor cell cycle and oxidative phosphorylation process and downregulate immune regulation, and Th17 and Th2 immune cell infiltration was increased in patients with lower expression of DNM3. Patients with a lower DNM3 expression had a higher rate of lymph node metastasis and poor prognosis. In summary, DNM3 is a tumor suppressor and an independent predictive factor of poor prognosis that regulates the cell cycle and immunosuppression in the tumor microenvironment in gastric cancer via methylation.

\section{Introduction}

Gastric cancer is a leading cause of cancer-related death worldwide. Currently, there is no effective early tumor marker, diagnostic method, and molecular therapeutic target for gastric cancer. With the development of genome sequencing technology, an increasing number of key driver genes and tumor suppressors have been discovered. However, some factors need to be clearly identified in gastric cancer.

Dynamin 3 (DNM3), a member of the dynamin (DNM) family, encodes a member of the guanosine triphosphate (GTP)-binding family that associate with microtubules and are involved in vesicular transport. ${ }^{1}$ DNM3 is widely expressed in cells of various tissues throughout the body. ${ }^{2,}{ }^{3}$ Given the important role of DNM3 in cell function, specific changes in DNM3 can lead to the development of a number of diseases. Researchers have found that genetic variability in DNM3 modifies the age of onset for LRRK2 Gly2019Ser parkinsonism in brain tissues. ${ }^{4}$ There are few studies on the role of the DNM family in malignancy, and the role of DNM3 in malignancy remains unclear. DNM3 has been suggested as a novel tumor suppressor; the methylation level of DNM3 is significantly increased in tumor tissues relative to that in normal control tissues, while the expression level is decreased. The expression level of DNM3 significantly correlates with the prognosis of tumors. ${ }^{5-7}$ The effect of DNM3 on the development of gastric cancer and its mechanisms are not yet known.

So, we conducted a systematic analysis of transcriptome, mutation and copy number alteration, methylation, gene set enrichment analysis (GSEA), gene ontology (GO), Kyoto Encyclopedia of Genes and Genomes (KEGG), immune infiltrates, immunohistochemical analysis (IHC), and long-term survival using the Cancer Genome Atlas Stomach Adenocarcinoma (TCGA-STAD) cohorts, and 160 patients of stomach 
adenocarcinoma in Fujian Medical University Union Hospital (FJMUUH) (48 quantitative PCR [qPCR] and 112 immunohistochemistry) to assess the prognostic significance of DNM3 gene expression in STAD.

\section{Materials And Methods}

\section{Patient datasets}

We analyzed TCGA-STAD cohorts. The original data were collected and downloaded from the TCGA (https://portal.gdc.cancer.gov/).

\section{Sample collection}

Gastric adenocarcinoma tissues were collected from 48 patients who had undergone gastrectomy and D2 lymph node dissection at the Department of Gastric Surgery, Fujian Medical University Union Hospital (FJMUUH) from January 2018 to July 2018. All the tissues were immediately stored in liquid nitrogen and preserved at $-80^{\circ} \mathrm{C}$. Paraffin blocks containing formalin-fixed tumor specimens collected from the other 112 patients at the FJMUUH between January 2013 and December 2015 were sliced into 4- $\mu \mathrm{m}$ serial sections and mounted on silane-coated glass slides for immunohistochemical analysis. All 160 patients were pathologically confirmed to have gastric adenocarcinoma after surgery without neoadjuvant chemotherapy or neoadjuvant radiotherapy. All samples were used in accordance with the principles of the Declaration of Helsinki and obtained informed consent from all the participants. The study was approved by the institutional review board of FJMUUH.

\section{Total RNA extraction and qPCR}

Total RNA from gastric cancer and paracancerous tissues was extracted using the Trizol kit (Invitrogen, Carlsbad, CA, USA) according to the manufacturer's instructions and used to obtain cDNA using Takara's reverse transcription system (Takara Bio Inc., Otsu, Shiga, Japan). qPCR was performed to detect the copy numbers of GAPDH and DNM3. GAPDH was used as an internal reference, and the $\Delta \triangle \mathrm{Ct}$ method was used for analysis. The primers used were as follows: human DNM3-Forward: 5'AGTTCGCCTTGAGATTGAAGC -3'; human DNM3-Reverse: 5'- CGTGTGGGGAATAGACTCGTAAA -3'; human GAPDH-Forward: 5'-GAAGGTGAAGGTCGGAGT-3'; human GAPDH-Reverse, 5'GAAGATGGTGATGGGATTTC-3'.

\section{Immunohistochemistry}

IHC-stained tissue sections were reviewed by two experienced pathologists and scored independently based on the intensity of cell staining and the positive ratio of the stained tumor cells. The primary antibodies used were rabbit polyclonal antibody against DNM3 (ProteinTech Group, Chicago, IL, USA). The percentage and intensity of DNM3-positive cells in randomly selected visual fields were evaluated. The staining scoring standards for DNM3 were as follows: 0 , no staining; 1, weak staining, light yellow; 2 , medium staining, yellowish-brown; and 3, strong staining, brown. The proportional score was as follows: $0, \leq 5 \%$ positive cells; $1,6 \%-25 \%$ positive cells; $2,26 \%-50 \%$ positive cells; and $3, \geq 51 \%$ positive cells 
(Figure 2). The IHC score was calculated by multiplying the intensity score with the proportional score, ranging from 0 to 9 . Patients with an $\mathrm{IHC}$ score $<3$ were classified into the low expression group. Otherwise, they were classified into the high expression group.

\section{Mutations and copy number alterations}

To detect mutations and copy number alterations in our study, the cBioPortal (https://www.cbioportal.org) and LinkedOmics (http://www.linkedomics.org) databases were used in TCGA-STAD with default parameters. The Wilcoxon text was used to detect the effect of mutations on DNM3 expression levels, and the Pearson correlation test was used to detect the effect of copy number alteration on DNM3 expression levels.

\section{Methylation status}

MethHC (http://MethHC.mbc.nctu.edu.tw) and LinkedOmics (http://www.linkedomics.org) databases were used for TCGA-STAD with default parameters.

\section{Correlation and enrichment analyses}

An online comprehensive network visual analytics platform for gene expression analysis (www.xiantao.love) was used for correlation analysis between DNM3 and other genes and GAES enrichment analyses. The DESeq2 $\mathrm{R}$ package was used for correlation analysis. ${ }^{8}$ The ClusterProfiler $\mathrm{R}$ package was used for GSEA with the following parameters: false discovery rate $<5 \%$, nominal $p$-value $<25 \%$, MSigDB collection enrichment (c2.cp.v7.2.symbols.gmt [Curated]). ${ }^{9,10} \mathrm{GO}$ and KEGG were performed based on all the genes in GSEA enrichment analysis using the ClusterProfiler R package (version 3.18.1).

\section{Immune cell infiltration}

The TIMER2 (http://timer.cistrome.org) database was used to assess the immune cell infiltration score of TCGA-STAD. We also used the online visual analytics platform (www.xiantao.love) for visualization (Box plot and Lollipop chart) based on the GSVA R package. ${ }^{11,12} \mathrm{~A}$ t-test was used to detect the enrichment score, and Spearman correlation was used for the molecular-immune cell correlation profile.

\section{Statistical analysis}

Standard statistical tests were used to analyze clinical data, including Student's t-test, Chi-square test, Fisher's exact test, and log-rank test. Fisher's exact test was used for categorical versus categorical variables; otherwise, the chi-square test was used. The Kruskal-Wallis test was used for categorical versus continuous variables to test whether any differences between subgroups were statistically significant; Spearman correlation was used for continuous variables. All statistical tests were two-sided and considered statistically significant when the $p$-value was $<0.05$. To account for multiple testing, $p$-values were adjusted using the Benjamini-Hochberg FDR correction. Kaplan-Meier plots were used to describe 
the overall survival (log-rank test). Univariate Cox proportional hazards regression models were used to identify variables associated with overall survival. Significant factors in the univariate analysis were further subjected to multivariate Cox regression analysis in a forward LR manner. All analyses of clinical data were performed using the Statistical Package for the Social Sciences (SPSS) version 22.0, for Windows (IBM, Chicago, IL, USA) or R (version 4.0.4).

\section{Results}

\section{DNM3 expression in STAD}

We first assessed DNM3 expression using STAD data from the TCGA database. We compared the expression levels with those in paired normal gastric tissues and found that DNM3 gene expression levels were significantly lower in STAD tissues $(p=0.006)$ than in paired normal tissues (Fig.1A). We further confirmed DNM3 expression by detecting the protein expression of DNM3 at the mRNA transcriptional level in 48 cases of fresh gastric cancer tissues and paired normal tissues using qRT-PCR in FJMUUH. DNM3 was found to be downregulated in 81.3\% (39/48) of gastric cancer tissues (Fig.1B-C).

\section{Mutations and copy number alterations of DNM3 in gastric cancer}

To assess the mutation and somatic copy number alteration (SCNA) of DNM3 in gastric cancer patients and its effect on DNM3 expression levels, we used cBioPortal and LinkedOmics databases to analyze the results. This showed that mutations resulted in decreased DNM3 expression levels (Log2FC=-1.423, $p=0.025$, Fig. 2A); SCNA had no significant relationship with DNM3 expression level ( $p=0.183$, Fig.2B). In contrast, only $16(4 \%)$ of 393 patients in the TCGA-STAD database had mutations and SCNA, including six missense mutations, one truncated mutation, eight copy number increases, and one copy number decrease (Fig. 2C), which may not explain the generally low DNM3 expression levels in gastric cancer patients.

\section{Methylation level of DNM3 in gastric cancer}

To assess the methylation level of DNM3 in gastric cancer patients, we used MethHC and LinkedOmics database analysis, which showed that the methylation level of DNM3 was higher in the gastric cancer group than in the normal group (Fig. 3A-B), and this high methylation level was associated with a poor prognosis in gastric cancer patients $(p=7.812 e-03)$ (Fig. 3C-D).

\section{Correlation and enrichment analyses}

To predict the function and associated pathways of DNM3, we performed a correlation analysis between DNM3 and other genes in gastric cancer using TCGA data (Extended Data Fig.1A-B). We performed GSEA using the entire DNM3-related gene dataset to identify signaling pathways that are differentially activated or inhibited in STAD (false discovery rate $<5 \%$, nominal p-value $<25 \%$ ) MSigDB collection enrichment (c2.cp.v7.2.symbols.gmt [curated]). We found that gene sets related to ribosome and oxidative phosphorylation were enriched in the low DNM3 gene expression phenotype, while immunoregulatory 
interactions between lymphoid and non-lymphoid cells were enriched in the high DNM3 gene expression phenotype (Fig. 4A-C). We further explored the GO analysis and KEGG pathway analysis based on all the genes in the GSEA enrichment analysis using the clusterProfiler $\mathrm{R}$ package. $\mathrm{GO}$ analysis revealed that low DNM3 expression was associated with the regulation of mitotic sister chromatid segregation, nucleartranscribed mRNA catabolic process, sister chromatid segregation, nuclear-transcribed mRNA catabolic process, and oxidative phosphorylation (Fig. 4D). The KEGG analysis revealed an enrichment and crosstalk in ribosomes and oxidative phosphorylation (Fig. 4E). These results suggest that low DNM3 expression is associated with cell mitosis and immunosuppression in the tumor microenvironment.

\section{Correlation between immune cell infiltration and DNM3 in gastric cancer}

To further validate the association between low DNM3 expression with immunosuppression in the tumor microenvironment, we used the TIMER2 (http://timer.cistrome.org/) database to assess the immune cell infiltration score of TCGA-STAD. We found that the Th17 cells, Th2 cells, and NK CD56dim cell infiltration levels were high in the low DNM3 expression group, while mast cells, NK cells, pDCs, B cells, follicular helper T cells (Tfh), effective memory T cells (Tem), T cells, central memory T cells (Tcm), Gammadelta T cells (Tgd), iDCs, and eosinophils showed a low DNM3 expression (Fig. 5).

\section{Correlation between IHC and clinical features}

We examined the expression level of DNM3 in 112 cases of gastric cancer and paired normal tissues using $\mathrm{IHC}$, and then scored the cells according to their staining intensity and percentage (Fig. 6A). We found that DNM3 was expressed at low levels in gastric cancer tissues compared to normal tissues (Fig. $6 \mathrm{~B}-\mathrm{C})$. Based on IHC scores, the patients were divided into two groups: high ( $\geq 3$ ) or low $(<3)$ DNM3 expression group. The association between DNM3 expression and clinical features in patients with gastric cancer is summarized in Table 1. Patients in the low DNM3 expression group had a higher rate of lymph node metastasis than those in the high expression group $(p=0.002)$, and the remaining factors were approximately equivalent $(p>0.05)$.

\section{Low expression of DNM3 is an independent risk factor for overall survival}

Cox regression analysis demonstrated that low DNM3 expression, as well as pT stage, $\mathrm{pN}$ stage, and pM stage were independent risk factors for 5 years OS in patients with gastric cancer $(p<0.05$, Extended Data Fig.2). The K-M curve showed that the 5-year overall survival (OS) rate of patients in the low DNM3 expression group was lower than that of patients in the high group ( $79.4 \%$ vs. $62.9 \%$, p<0.05; Fig. 6D).

\section{Discussion}

DNM3 is a novel tumor suppressor with limited studies. How it exerts its suppressor effect and what causes its diminished suppressor effect remain unclear. Based on our study and analysis of public databases, we found that DNM3 methylation status was increased in gastric cancer tissues, leading to downregulation of DNM3 expression. Low DNM3 expression weakened cell cycle regulation, enhanced 
oxidative phosphorylation, and suppressed immune regulation, ultimately leading to a poor prognosis in patients with low DNM3 expression.

In this study, we found that DNM3 has low mutation and SCNA rates in patients with gastric cancer; however, we observed a high methylation status in these patients, which affected the expression level of the DNM3 gene. DNM3 hypermethylation was also found in tumor tissues such as liver cancer and breast cancer, $^{6-7,13}$ indicating that the suppressor gene DNM3 may cause a decrease in the expression level of DNM3 mRNA due to promoter methylation. This is also evidenced by the fact that methylation inhibitors can reverse the expression levels of DNM3.

Functional enrichment analysis revealed that reduced DNM3 levels led to a slowing down of mitosis and a weakening of its monitoring role, with enrichment of genes related to $G 1, S, G 2$, and $M$ phases, and enhanced transcriptional-translational processes. Further experiments are needed to investigate whether cell cycle regulation by DNM3 is dependent on p53. It is known that an important function of DNM3 may be related to the nitric oxide (NO) pathway. ${ }^{14} \mathrm{Cao}$ et al. found that the proline-rich $\mathrm{COOH}$ terminal domain in DNM2 interacts with the FAD region in nitric oxide synthase 3 (NOS3) to activate NOS3 and promote NO production. ${ }^{15}$ In addition, p53, which is activated by NOS, induces cell cycle arrest and promotes apoptosis of hepatocellular carcinoma cells. ${ }^{16}$

We also found that a reduced level of DNM3 expression decreased the immunomodulatory effect between lymphocytes and non-lymphocytes. Major immune effector cells (mast cells, NK cells, B cells, and $T$ cells) have a reduced enrichment fraction in the tumor microenvironment compared to Th17 and Th2 cells. The mechanism of Th17 cells in tumor immunity is unclear, but some studies have found that IL-17 can promote tumor growth. ${ }^{17-19}$ The tumor microenvironment is in a state of Th2 dominant differentiation, and the cytokines they produce have an inhibitory effect on the proliferation and differentiation of Th1 cells and cytotoxic T lymphocytes. This leads to a weakening of the body's antitumor immune function and allows tumor cells to escape from immune surveillance and immune attack. $^{20-22}$

In summary, DNM3 is a tumor suppressor and an independent predictive factor of poor prognosis that regulates the cell cycle and immunosuppression in the tumor microenvironment in gastric cancer via methylation.

\section{Declarations}

Ethics approval and consent to participate: The study was approved by IRB of Fujian Medical University Union Hospital. All samples were used in accordance with the principles of the Declaration of Helsinki and obtained informed consent from all the participants.

Consent for publication: This article doesn't report an individual participant's data in any form. 
Availability of data and materials: Databases and software used for bioinformatics analysis were described and referenced in the respective Method Details subsections and listed in the Key Resources Table (Extended Data Table 1). Other data can be available on request through contacted to hcmlr2002@163.com.

Competing interests: I confirm that I have read journal's guidance on competing interests and have included a statement indicating that none of the authors have any competing interests in the manuscript.

Funding: The study was sponsored by the Scientific and Technological Innovation Joint Capital Projects of Fujian Province (2019Y9098). The general project of sailing fund of Fujian Medical University (2018QH1021).

Authors' contributions: Conception/Design: Huang $\mathrm{CM}$, Zheng $\mathrm{CH}$, Tu RH, Lin JX; Collection and/or assembly of data: Tu RH, Lin JX,Chen QY, Li P, Xie JW, Wang JB, Lin JX, Lu J, Cao LL, Lin M, Huang ZN, Lin JL, Zheng HL, Zheng $\mathrm{CH}$, and Huang CM; Data analysis and interpretation: Tu RH, Lin JX and Li P; Manuscript writing: Tu RH, Lin JX, Li P, Zheng CH, and Huang CM.

\section{Acknowledgements: Not applicable}

\section{References}

1. Shpetner H S, Vallee R B. Identification of dynamin, a novel mechanochemical enzyme that mediates interaction setween microtubules. Cell, 1989. 59(3): p. 421-432.

2. Ferguson, S.M., et al., A Selective Activity-Dependent Requirement for Dynamin 1 in Synaptic Vesicle Endocytosis. Science, 2007. 316(5824): p. 570-574.

3. Tanifuji, S. and S. Mochida, Contribution of dynamin isoforms to synaptic vesicle recycling in sympathetic neurons. Neuroscience Research, 2011. 71(4): p. e217-e217.

4. Trinh Joanne, Gustavsson Emil K, Vilariño-Güell Carles, et al. DNM3 and genetic modifiers of age of onset in LRRK2 Gly2019Ser parkinsonism: a genome-wide linkage and association study. Lancet Neurol, 2016, 15: 1248-1256.

5. Shen Jing, Wang Shuang, Zhang Yu-Jing, et al. Genome-wide DNA methylation profiles in hepatocellular carcinoma. Hepatology, 2012, 55: 1799-808.

6. Inokawa Yoshikuni, Nomoto Shuji, Hishida Mitsuhiro, et al. Dynamin 3: a new candidate tumor suppressor gene in hepatocellular carcinoma detected by triple combination array analysis. Onco Targets Ther, 2013, 6: 1417-24.

7. Mao Xiao-Hong, Ye Qiang, Zhang Guo-Bing et al. Identification of differentially methylated genes as diagnostic and prognostic biomarkers of breast cancer. World J Surg Oncol, 2021, 19: 29.

8. Love M I, Huber W, Anders S. Moderated estimation of fold change and dispersion for RNA-seq data with DESeq2. Genome biology, 2014, 15(12): 550. 
9. Yu G, Wang L G, Han Y, et al. clusterProfiler: an R package for comparing biological themes among gene clusters. Omics: a journal of integrative biology, 2012, 16(5): 284-287.

10. Subramanian A, Tamayo P, Mootha V K, et al. Gene set enrichment analysis: a knowledge-based approach for interpreting genome-wide expression profiles[J]. Proceedings of the National Academy of Sciences, 2005, 102(43): 15545-15550.

11. Hänzelmann, Sonja, Robert Castelo, Justin Guinney. GSVA: gene set variation analysis for microarray and RNA-seq data. BMC bioinformatics 14.1 (2013): 1-15.

12. Bindea Gabriela, Mlecnik Bernhard, Tosolini Marie, et al. Spatiotemporal dynamics of intratumoral immune cells reveal the immune landscape in human cancer. Immunity, 2013, 39: 782-95.

13. Uehiro Natsue, Sato Fumiaki, Pu Fengling, et al. Circulating cell-free DNA-based epigenetic assay can detect early breast cancer. Breast Cancer Res, 2016, 18: 129.

14. Cao, H., F. Garcia, and M.A. Mcniven. Differential Distribution of Dynamin Isoforms in Mammalian Cells. Mol.biol.cell, 1998. 9(9): p. 2595-2609.

15. Cao, S., J. Yao, and V. Shah. The proline-rich domain of dynamin-2 is responsible for dynamindependent in vitro potentiation of endothelial nitric-oxide synthase activity via selective effects on reductase domain function. Journal of Biological Chemistry, 2003. 278(8): p. 5894-901.

16. Lim Shuhui, Hung Amos C, Porter Alan G. Focused PCR screen reveals p53 dependence of nitric oxide-induced apoptosis and up-regulation of maspin and plasminogen activator inhibitor-1 in tumor cells. Mol Cancer Res, 2009, 7: 55-66.

17. Martin-Orozco Natalia, Muranski Pawel, Chung Yeonseok, et al. T helper 17 cells promote cytotoxic $T$ cell activation in tumor immunity. Immunity, 2009, 31: 787-98.

18. Wang Lin, Yi Tangsheng, Kortylewski Marcin, et al. IL-17 can promote tumor growth through an IL-6Stat3 signaling pathway. J Exp Med, 2009, 206: 1457-64.

19. Nam Jeong-Seok, Terabe Masaki, Kang Mi-Jin, et al. Transforming growth factor beta subverts the immune system into directly promoting tumor growth through interleukin-17. Cancer Res, 2008, 68: 3915-23.

20. Thakur Archana, Schalk Dana, Sarkar Sanila H, et al. A Th1 cytokine-enriched microenvironment enhances tumor killing by activated T cells armed with bispecific antibodies and inhibits the development of myeloid-derived suppressor cells. Cancer Immunol Immunother, 2012, 61: 497-509.

21. Pradhan Pallab, Qin Hong, Leleux Jardin A et al. The effect of combined IL 10 siRNA and CpG ODN as pathogen-mimicking microparticles on Th1/Th2 cytokine balance in dendritic cells and protective immunity against B cell lymphoma. Biomaterials, 2014, 35: 5491-504.

22. Block Matthew S, Nevala Wendy K, Leontovich Alexey A, et al. Differential response of human and mouse dendritic cells to VEGF determines interspecies discrepancies in tumor-mediated TH1/TH2 polarity shift. Clin Cancer Res, 2011, 17: 1776-83.

\section{Tables}


Table 1. The association between DNM3 expression and clinical features of patients with gastric cancer in FJMUUH 
Variables

Total

DNM3 expression

P

Low ( $n=79)$

High(n=33)

Gender

0.121

Male

88

59

29

Female

24

20

4

Age at surgery(years)

0.172

$\$ 60$

75

56

19

$\leq 60$

37

23

14

Size of primary tumor (cm)

\begin{tabular}{|c|c|c|c|c|}
\hline$\otimes 5$ & 46 & 31 & 15 & \\
\hline$\leq 5$ & 66 & 48 & 18 & \\
\hline $\begin{array}{l}\text { Location of primary } \\
\text { tumor }\end{array}$ & & & & 0.418 \\
\hline Lower $1 / 3$ & 49 & 35 & 14 & \\
\hline Middle 1/3 & 23 & 18 & 5 & \\
\hline Upper $1 / 3$ & 37 & 25 & 12 & \\
\hline More than $1 / 3$ & 3 & 1 & 2 & \\
\hline Degree of differentiation & & & & 0.862 \\
\hline Well/moderate & 36 & 25 & 11 & \\
\hline Poor and not & 76 & 54 & 22 & \\
\hline Depth of invasion & & & & 0.996 \\
\hline $\mathrm{T} 1+\mathrm{T} 2$ & 17 & 12 & 5 & \\
\hline $\mathrm{T} 3+\mathrm{T} 4$ & 95 & 67 & 28 & \\
\hline Lymph node metastasis & & & & 0.002 \\
\hline NO & 14 & 5 & 9 & \\
\hline N1-3 & 98 & 74 & 24 & \\
\hline TNM stage & & & & 0.132 \\
\hline$\nabla+\square$ & 36 & 22 & 14 & \\
\hline$\nabla+\square$ & 76 & 57 & 19 & \\
\hline
\end{tabular}


Distant metastasis

0.957

\begin{tabular}{lllll} 
Negative & 105 & 74 & 31 & \\
\hline Positive & 7 & 5 & 2 & \\
\hline CEA [ug/L, Median(IQR)] & $2.80(1.70-3.90)$ & $2.80(1.70-3.90)$ & $2.82(1.73-3.88)$ & 0.753 \\
\hline CA199 [kU/L, & $9.51(4.72-17.02)$ & $9.07(4.31-14.74)$ & $9.92(5.87-20.19)$ & 0.555 \\
Median(IQR)] & & & & 0.796 \\
\hline CA125 [kU/L, & $10.81(7.34-$ & $10.82(7.13-$ & $10.52(7.82-$ & \\
Median(IQR)] & $14.41)$ & $14.41)$ & $16.27)$ & \\
\hline AFP [mg/mL, & $2.82(1.97-4.54)$ & $2.76(1.87-4.47)$ & $3.11(2.20-6.07)$ & 0.359 \\
Median(IQR)] & & & & \\
Figures & & & & \\
\hline
\end{tabular}




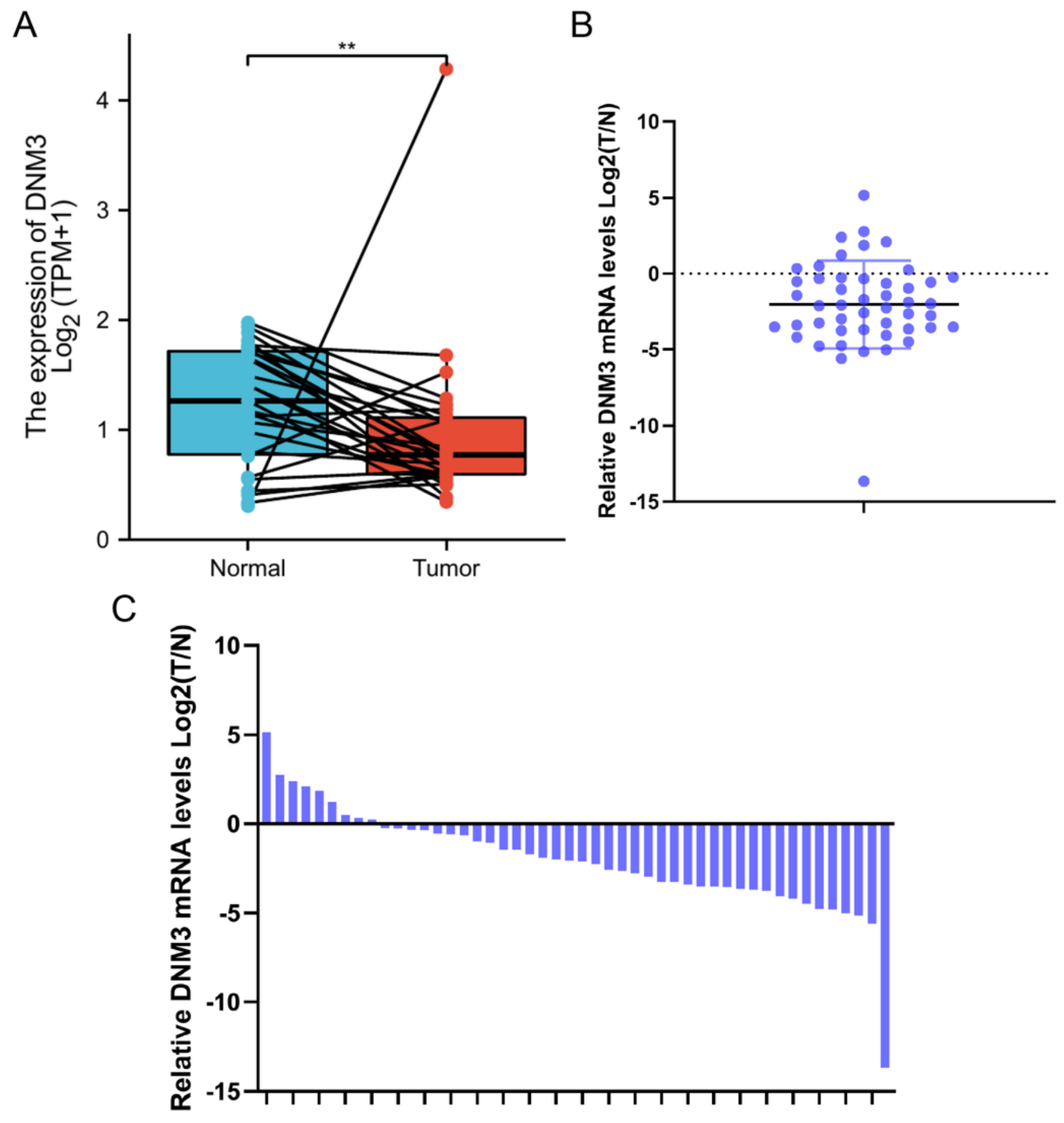

Figure 1

DNM3 expression in stomach adenocarcinoma tissues. (A) DNM3 expression in tumor and paired normal tissues in TCGA-STAD. (B-C) qRT-PCR analysis of DNM3 expression in 48 cases of gastric cancer in Fujian Medical University Union Hospital (FJMUUH). 

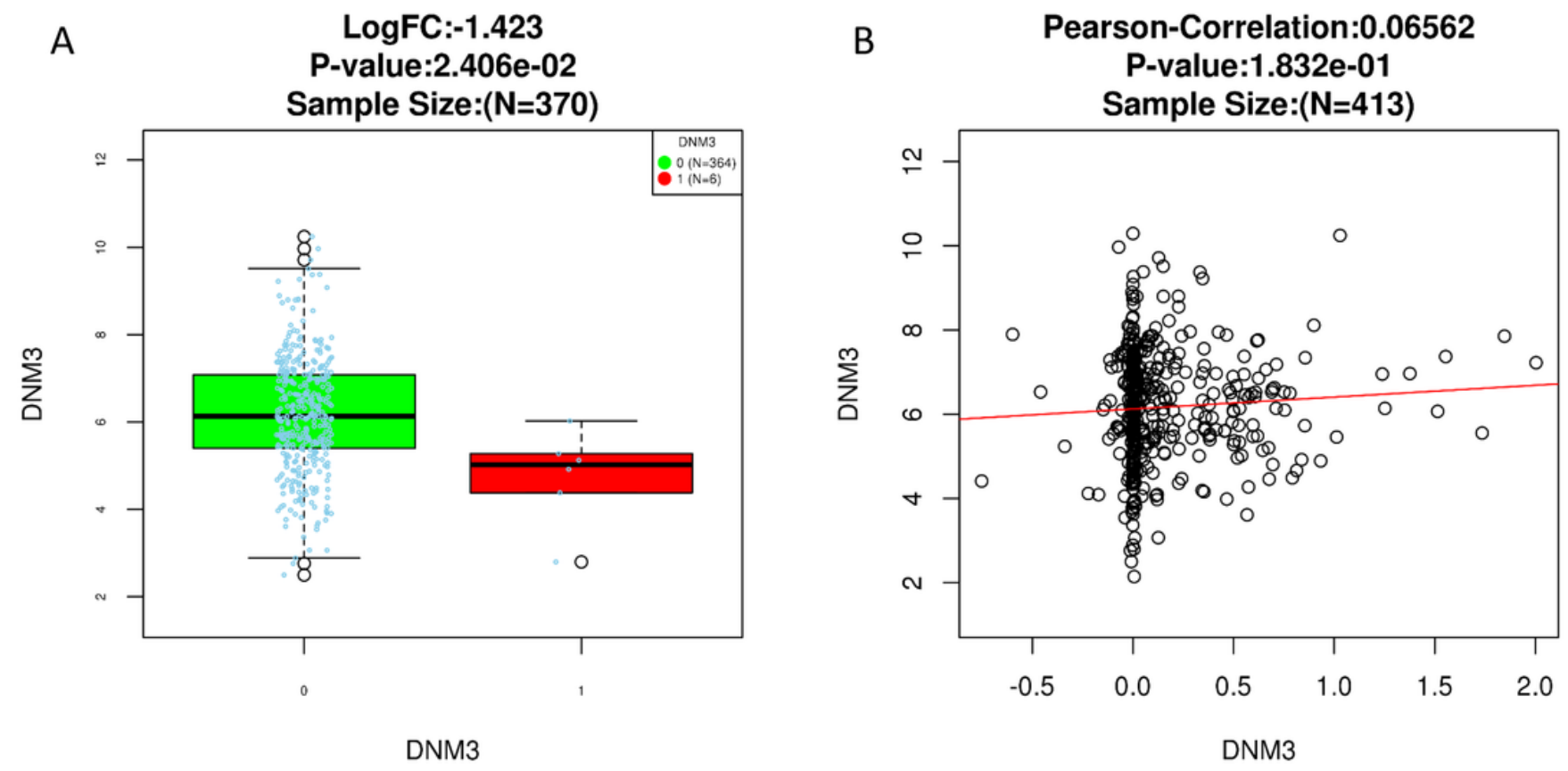

C

onмs

Genetic Alteration

Missense Mutation (unknown significance) - Truncating Mutation (unknown significance)

Amplification (unknown significance)

Deep Deletion (unknown significance) No alterations

Figure 2

Mutation and somatic copy number alteration (SCNA) of DNM3 in TCGA-STAD and its effect on DNM3 expression levels via the cBioPortal and LinkedOmics database. (A) Effect of DNM3 mutations on DNM3 expression levels. (B) Effect of DNM3 SCNA on DNM3 expression levels. (C) Mutation and SCNA of DNM3 in TCGA-STAD. 
A

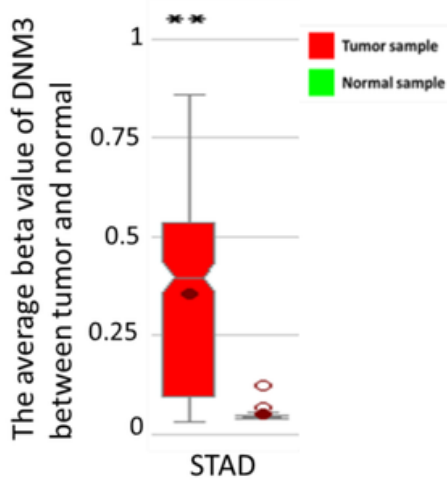

C

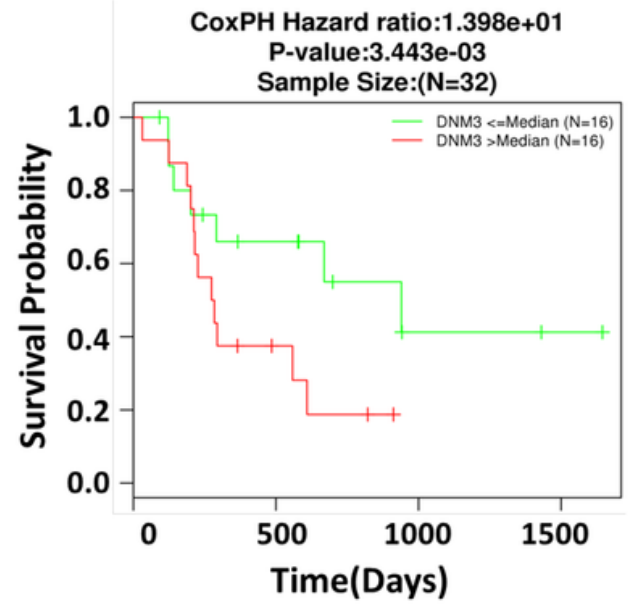

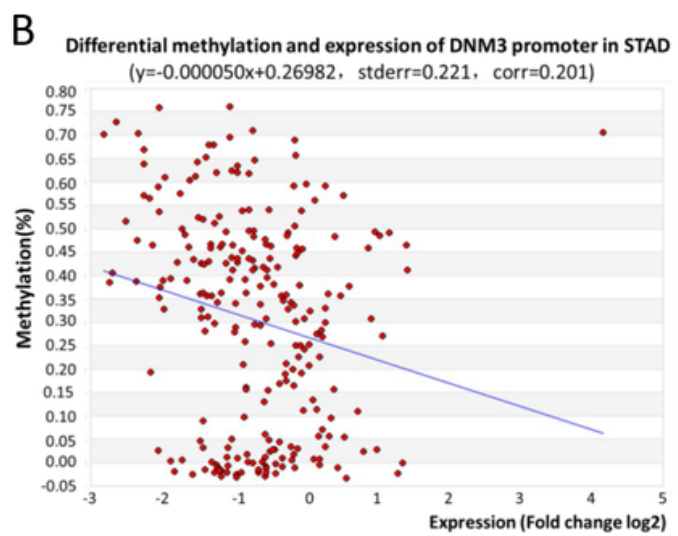

D overall_survival Association Result

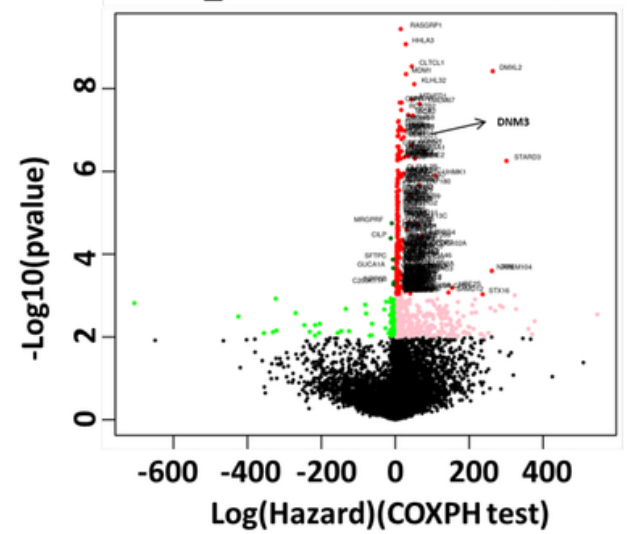

\section{Figure 3}

Methylation level of DNM3 in gastric cancer using MethHC and LinkedOmics database. (A) The average beta value of DNM3 between tumor and normal tissue. (B) Differential methylation and expression of DNM3 promoter in STAD. (C) Kaplan-Meier curve for overall survival in high/low DNM3 methylation patients with gastric cancer. (D) Volcano plot of association between overall survival and differential methylation genes. 

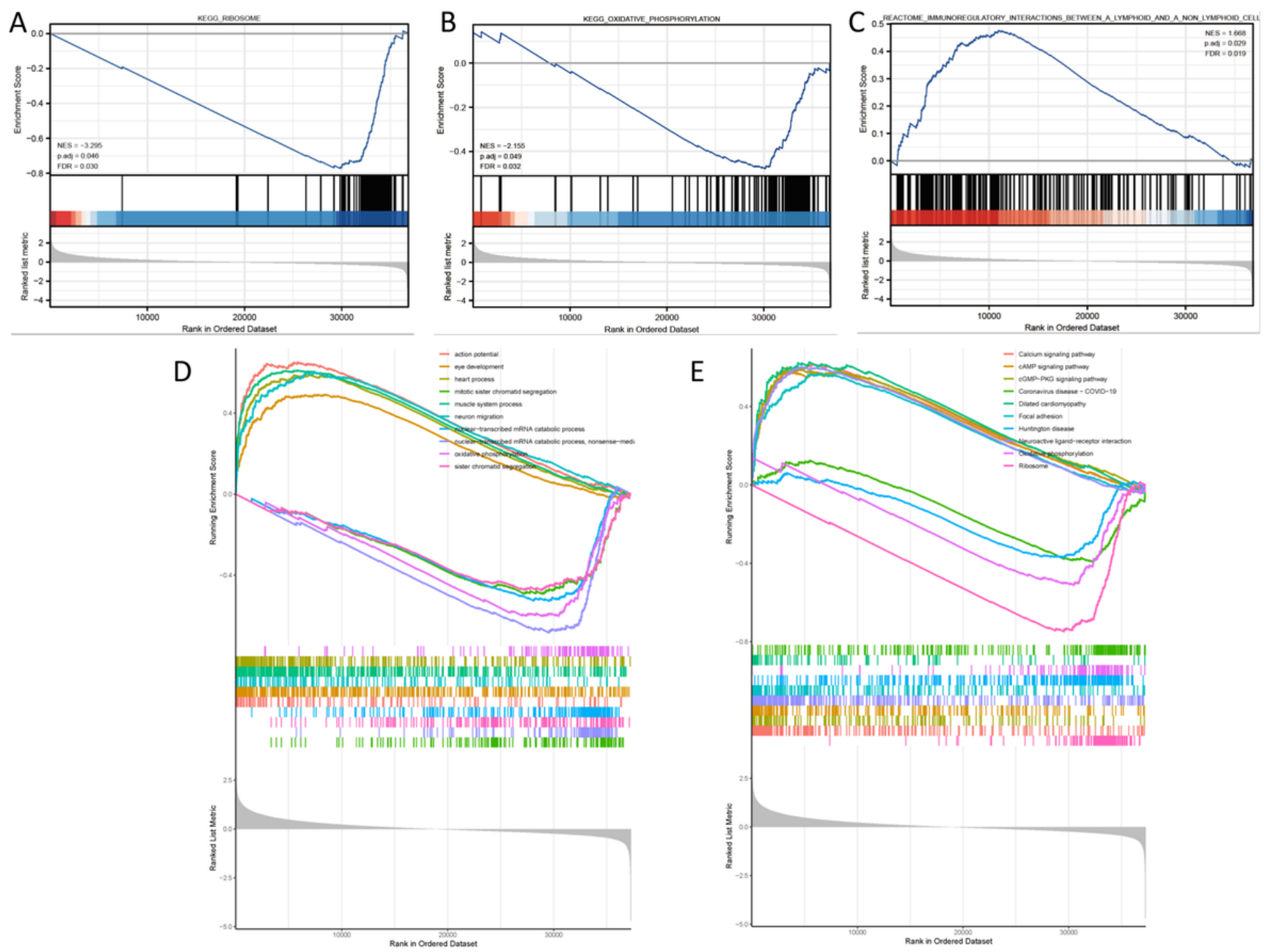

$E$

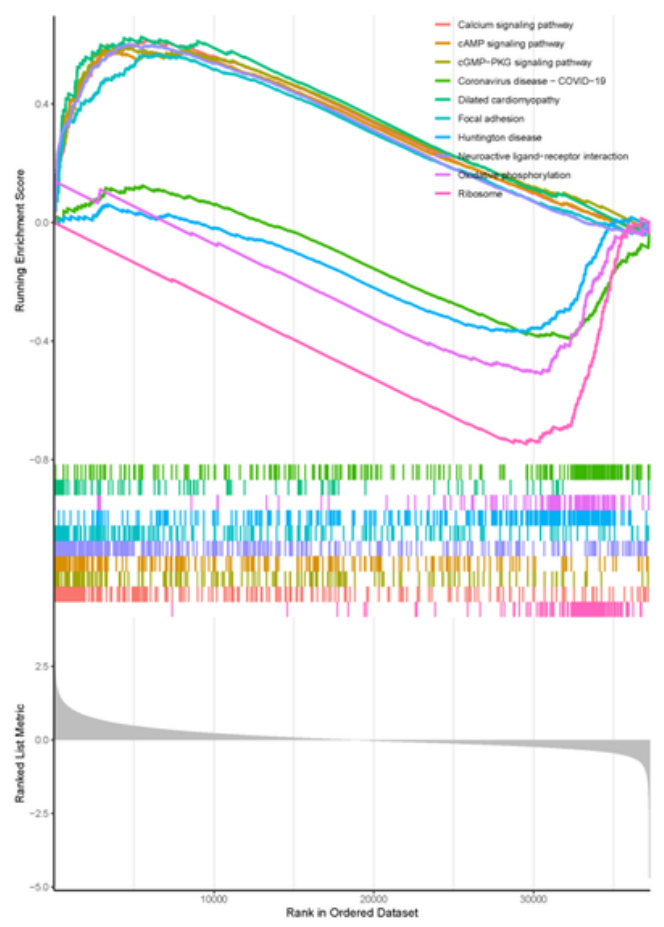

Figure 4

Pathway enrichment and function analyses of DNM3 in gastric cancer. (A-C) Gene set enrichment plots of (A) ribosome, (B) oxidative phosphorylation, (C) immunoregulatory interactions between a lymphoid and a non-lymphoid cell. (D) Top 10 significant Gene Ontology terms associated with DNM3. (E) Top 10 significant KEGG pathways associated with DNM3. 

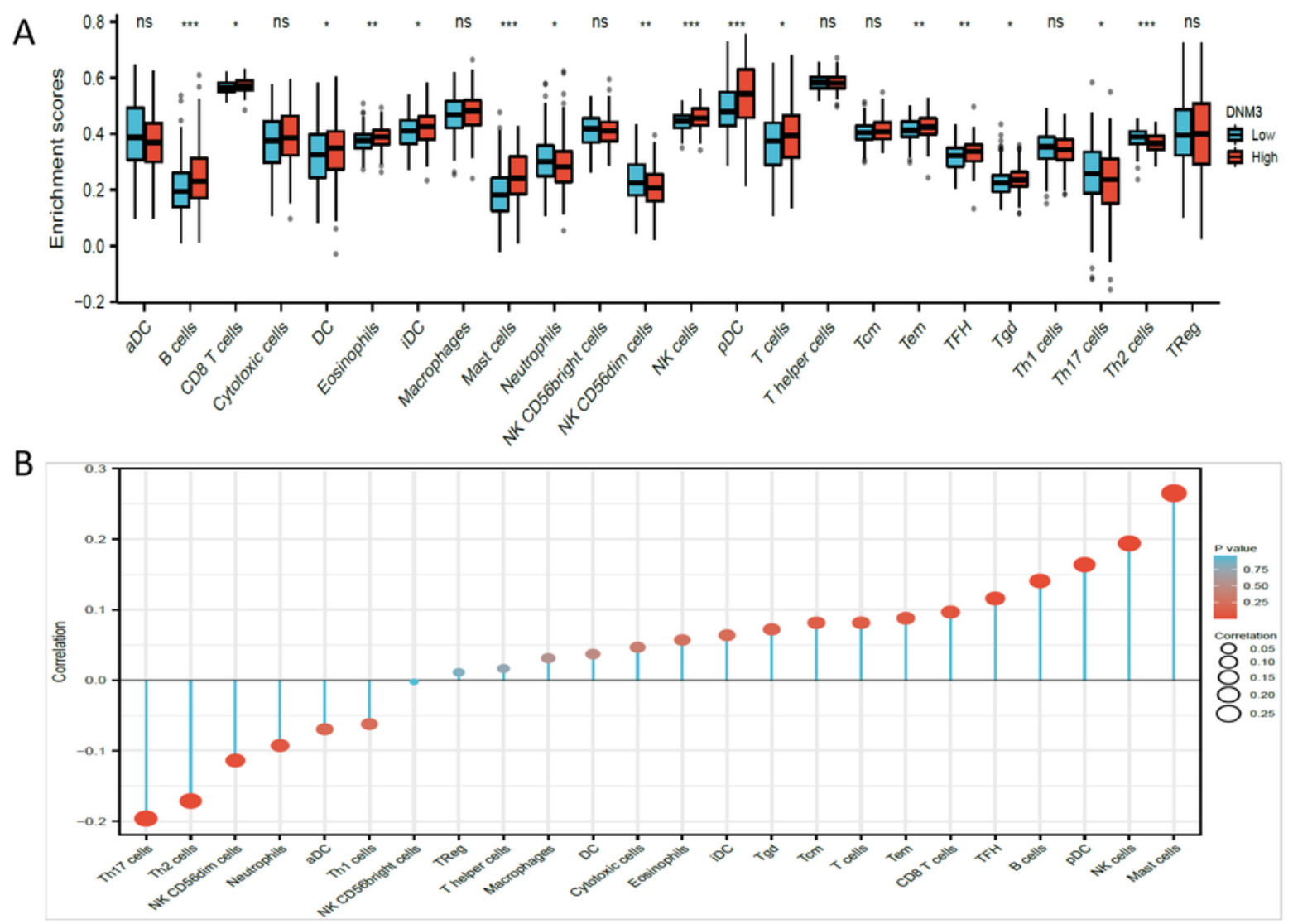

Figure 5

Correlation between immune cell infiltration and DNM3 in gastric cancer. (A) Box plot of immune cell infiltration level in high and low DNM3 expression group in TCGA-STAD. (B) Lollipop chart of immune cell infiltration level in high and low DNM3 expression groups sorted by correlation. 
A

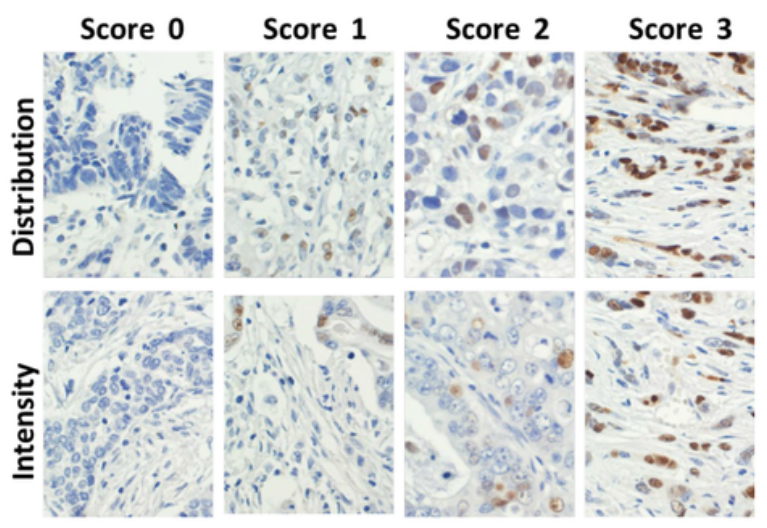

B



C

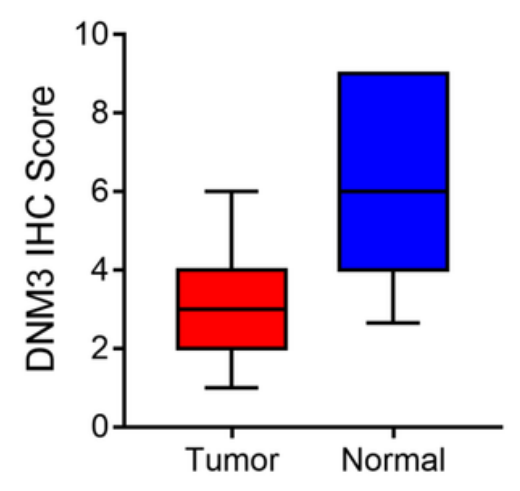

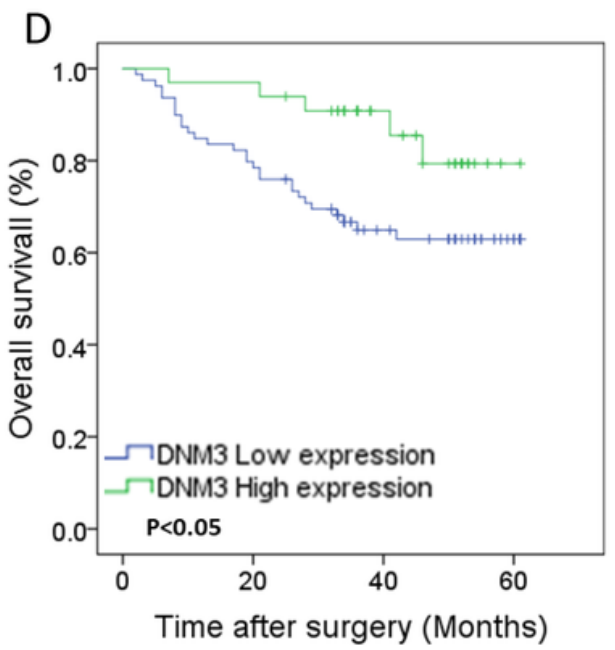

Figure 6

Association between DNM expression and cancer patient prognosis. (A) Immunohistochemical (IHC) score of DNM3. (B) IHC score in tumor (high) and paired normal tissues (low). (C) Box plot evaluating DNM3-IHC score of patients with gastric cancer in FJMUUH. (D) Kaplan-Meier curve for overall survival in high/low DNM3 expression patients with gastric cancer.

\section{Supplementary Files}

This is a list of supplementary files associated with this preprint. Click to download.

- SupplementalFiles.docx 